\title{
Epithelioid trophoblastic tumour: a case report and review of the literature
}

\author{
P M L H Vencken, P C Ewing, R P Zweemer
}

Epithelioid trophoblastic tumour (ETT) is an unusual type of trophoblastic tumour, which can cause difficulties in diagnosis and (as a consequence) in treatment. The literature suggests that surgery should be the treatment of choice for ETT as it is not responsive to chemotherapeutic agents, used in the treatment of other types of gestational trophoblastic diseases. This case report describes an ETT, which was initially diagnosed as a carcinoma of the cervix. Surgical management was chosen based on the literature. 6 months later the patient also developed a plasmacytoma and was treated with radiotherapy. The occurrence of ETT and plasmacytoma in combination has never been described before. This case report describes a rare case of an atypical trophoblastic tumour, with problematic differential diagnosis. Treatment of carcinoma of the cervix would have necessitated postoperative radiotherapy, but on diagnosis of ETT, surgical management was considered sufficient. Hence, it is important to consider the occurrence of ETTs, although rare, in patients with atypical cervical or endometrial cancer, and in patients diagnosed with a gestational trophoblastic tumour, who do not respond to appropriate chemotherapy.

G estational trophoblastic disease (GTD) includes complete and partial hydatiform mole, invasive mole, choriocarcinoma, placental site trophoblastic tumour and epithelioid trophoblastic tumour (ETT). ${ }^{12}$ ETT is an unusual type of trophoblastic tumour, which on microscopy resembles a carcinoma. ${ }^{13}$ This can potentially lead to difficulties in diagnosis. The tumour was originally named "atypical choriocarcinoma", because the first descriptions of the disease were metastases of the lung in patients who had received chemotherapy for what was thought to be choriocarcinoma. ${ }^{45}$ These patients were chemoresistant, but subsequent surgical resection seemed curative, making choriocarcinoma unlikely. ${ }^{2}$

The term "epithelioid trophoblastic tumour" was first proposed in 1994. ${ }^{4}$ It was suggested then that the development of an ETT was the result of chemotherapy given for choriocarcinoma, and that chemotherapy had influenced the growth of tumour cells by drug-induced changes. ${ }^{3}$ However, in 1998, Shih et al ${ }^{6}$ reported 14 patients with ETT who had no history of chemotherapy, suggesting that ETT is a distinct pathological entity and not simply a treatment-related finding. ${ }^{3}$

\section{CASE REPORT}

A 43-year-old woman was referred to a gynaecologist with an 8-month history of secondary amenorrhoea and vaginal discharge. Flushes were absent. Her obstetric history showed an abortion and a twin pregnancy, resulting in a normal delivery 13 years before presentation. Her medical history showed hypertension.

\section{Gynaecological examination}

Gynaecological examination showed abundant turbid mucus in the absence of an apparent abnormality of the cervix.
Recto-vaginal examination was unremarkable. Transvaginal ultrasound showed a hypodense abnormality next to the uterus, which could not be explained. A cervical smear showed no abnormal cells.

Bacteriological cultures showed the presence of haemolytic group B streptococcus. Analysis of hormone levels (folliclestimulating hormone, luteinising hormone, oestrogen, prolactine, thyroid-stimulating hormone and $\beta$ human chorionic gonadotropin (HCG) hormone $(<2.0 \mathrm{IU} / \mathrm{l}))$ and the erythrocyte sedimentation rate were normal. A pyometra was diagnosed during curettage and, as the finding of the pus could not be explained, a laparotomy was performed instantly. The ovary, fallopian tubes and corpus uteri were normal. A soft tumour was seen at the isthmus, and the cervix was strikingly long (8$10 \mathrm{~cm})$. The uterus was removed.

\section{Pathology}

A tumour was present in the hysterectomy specimen, which was initially interpreted as a glassy cell carcinoma (a variant of adenosquamous carcinoma) of the cervix.

As part of the work-up for adjuvant radiotherapy, the slides were reviewed and a diagnosis of ETT was suggested. The histological appearances were of a tumour comprising cells with pleomorphic nuclei and relatively abundant eosinophilic cytoplasm lying in sheets and nests. Hyaline material was present between the tumour cells (fig 1). In some areas, the cells with lesions had slightly smaller, more oval nuclei and sometimes a clearer cytoplasm. Particularly striking were areas of "geographical necrosis" (fig 2). Also of note was a relatively low mitotic count when compared with the degree of cytological atypia. Although there were some regions at the periphery of the tumour with 7 mitotic cells per 10 high-power fields, mitoses were scarce in most of the tumour. The tumour had a well-circumscribed margin, around which a lymphocytic infiltrate was present.

Immunohistochemical staining showed positivity with epithelial markers (cytokeratin 7, epithelial membrane antigen and cell adhesion molecule 5.2). In addition, there was positive staining with inhibin in some regions of the tumour. Placental alkaline phosphatase was focally positive, human placental lactogen was positive in occasional cells and HCG was negative. P63 (Dako 4A4 clone, Dako, Rotterdam, The Netherlands) was positive in most of the tumour cell nuclei. ETT was diagnosed.

The slides were submitted to another academic centre for review, where the diagnosis was confirmed. In situ hybridisation showed a Y-chromosome in the tumour cells, further supporting the gestational trophoblastic origin of the tumour.

\section{Follow-up}

Computed tomography did not show distant metastases or enlarged lymph nodes. An expectative policy was followed. One month after surgery, the $\beta$-HCG was $<0.5$ IU/l.

Abbreviations: ETT, epithelioid trophoblastic tumour; GTD, gestational trophoblastic disease; HCG, human chorionic gonadotropin 
Six months after surgery, the patient had pain in the right hip, buttock and inguinal region. Computed tomography showed a lesion in the right acetabulum. A bone scan showed an abnormality in the right acetabulum, extending into the right sacro-iliac joint and affecting the right trochanter. These findings were initially interpreted as metastasis, but a bone biopsy showed atypical plasma cell proliferation. A monoclonal $\gamma$-immunoglobulin $\kappa$ peak was found in the protein spectrum. Plasmacytoma was diagnosed. The patient was treated with 23 fractions of 2 Gy radiotherapy. A year after hysterectomy and 6 months after radiotherapy, the patient is in complete remission for both malignancies.

\section{DISCUSSION}

ETTs are rare. ${ }^{5}$ Only 35 cases have been reported (in 13 papers), including tumours termed atypical choriocarcinoma and nodules of intermediate trophoblast. ${ }^{2}$ The age of patients who develop ETT was reported to range from 15 to 48 (mean 36.1) years, with only one reported patient being a postmenopausal woman. ${ }^{2}$

In most of the patients, as in our patient, the previous gestational event was a full-term delivery (67\%). Miscarriages $(16 \%)$ and hydatiform moles have also been described as antecedent gestational events. ${ }^{1}$

The reported interval between the previous gestation and the diagnosis ranged from 1 to 18 (mean 6.2) years. ${ }^{1-35}$ In our patient the interval was 13 years, which was compatible with the findings described in the literature.

Abnormal vaginal bleeding is the most commonly presenting symptom. ${ }^{12}$ Our patient presented with atypical amenorrhoea and vaginal discharge: amenorrhoea has not previously been described as a symptom of ETT. $\beta$-HCG levels in the serum are almost always slightly raised, but this was not the case in our patient. ${ }^{6}$

In a study of 14 ETTs, ${ }^{23} 30 \%$ were located in the uterine corpus, $50 \%$ in the lower uterine segment or endocervix (as in our patient) and the remaining $20 \%$ at extrauterine sites (small bowel and lungs). ${ }^{25}$ Tumour sizes varied from 0.5 to $4.0 \mathrm{~cm} .{ }^{1}$ All the tumours were solitary, discrete nodules deeply invading the cervix or myometrium. ${ }^{1}$ The lesions were typically tan to brown, with varying amounts of haemorrhage and necrosis.

Microscopically, the tumour is typically composed of a population of mononucleate cells, forming nests and solid masses and resembling the cells of the intermediate trophoblast in the chorion leave. ${ }^{1-3}{ }^{6}$ ETTs are well circumscribed, with a surrounding lymphocytic infiltrate, as in our patient. The tumour we describe also shows the typical extensive necrosis creating a geographical pattern. ${ }^{16}$ This pattern is sometimes accompanied by dystrophic calcification.

On immunohistochemical staining, the tumour was typically positive for cytokeratin, epithelial membrane antigen and inhibin. Human placental lactogen, HCG, placental alkaline phosphatase and Mel-cell adhesion molecule were expressed, but only focally. ${ }^{6}$ The Ki-67 labelling index was $10-25 \%$.

P63 is positive in most ETTs and can be particularly useful when the differential diagnosis is with placental site trophoblastic tumour. ${ }^{7}$

In our patient, the tumour was initially diagnosed as a glassy cell carcinoma of the cervix. Differentiating an ETT from a carcinoma of the cervix can be difficult. This is partly because of the tendency of ETTs to grow in the lower uterine segment and cervix. ${ }^{126}$ The nests and cords of tumour cells have an epithelioid appearance and the eosinophilic extracellular matrix that accompanies the tumour can resemble keratin, heightening the similarity of the lesion to squamous cell carcinoma of the cervix. ${ }^{5}$ However, keratin pearls and intercellular bridges, which can be seen in squamous cell carcinoma, are not present in ETT. ${ }^{23}$ Results of immunohistochemical staining can help to differentiate between the two tumours. ${ }^{13}$ HCG levels are generally raised in ETT, although this was not the case in our patient ${ }^{6}$ and thus this screening marker is not reliable in differentiating cervical carcinoma.

These problems in differential diagnosis may imply that these lesions have been under-reported in the literature. ${ }^{6}$ The differential diagnosis is important with respect to therapeutic decision making: our patient would have undergone irradiation if ETT had not been diagnosed.

Only 35 cases of ETT have been reported; hence, it is difficult to identify any features that may predict the outcome. From the published series, metastasis and death occur in $25 \%$ and $10 \%$ of patients, respectively. ${ }^{1}$ ETTs do not respond to the chemotherapeutic agents used in the treatment of other types of GTD. ${ }^{18}$ If a patient suspected of having a gestational trophoblastic tumour is unresponsive to chemotherapy, further histological examination should be carried out and the possibility of an ETT should be considered. ${ }^{4}$ Hysterectomy and lung resection have been used successfully. ${ }^{1}$

In summary, we report an ETT with atypical presentation. Initial histological examination suggested that it was a carcinoma of the cervix, but the diagnosis of ETT was later confirmed by further microscopical investigation and immunohistochemical studies. This diagnosis allowed the appropriate choice of surgical treatment without irradiation. As far as we know, this is the first case of a patient with an ETT, who later developed a plasmacytoma. We have found no description in the literature of a link between GTD and plasma cell neoplasm.

\section{ACKNOWLEDGEMENTS}

We thank R Rouse, Department of Pathology, Middelburg, The Netherlands, P Bult, Department of Pathology, University Hospital Nijmegen, The Netherlands, and H C van Doorn, Department of Gynecology, Erasmus MC Rotterdam, The Netherlands.

\section{Authors' affiliations \\ P M L H Vencken, R P Zweemer, Department of Obstetrics and Gynecology, Erasmus MC University Medical Centre, Rotterdam, The Netherlands \\ P C Ewing, Department of Pathology, Erasmus MC University Medical Center}

Competing interests: None.

Correspondence to: P M L H Vencken, Department of Obstetrics and Gynecology, Erasmus MC University Medical Center, Drive Molewaterplein 60, PO Box 3015 GJ, Rotterdam, The Netherlands; p.vencken@erasmusmc.nl

Accepted for publication 22 July 2005

\section{REFERENCES}

1 Kurman RJ. Blaustein's pathology of the female genital tract. 5th edn. 2002, (Chapter 24).

2 Coulson LE, Kong CS, Zaloudek C. Epithelioid trophoblastic tumor of the uterus in a postmenopausal woman: a case report and review of the literature. Am J Surg Pathol 2000;24:1558-62.

3 Kuo KT, Chen MJ, Lin MC. Epithelioid trophoblastic tumor of the broad ligament: a case report and review of the literature. Am J Surg Pathol 2004;28:405-9.

4 Shen D, Khoo US, Ngan HYS, et al. Coexisting epithelioid trophoblastic tumor and choriocarcinoma of the uterus following a chemoresistant hydatiform mole. Arch Pathol Lab Med 2003;127:291-3.

5 Meydanli MM, Kucukali T, Usubutun A, et al. Epithelioid trophoblastic tumor of the endocervix: a case report. Gynecol Oncol 2002;87:210-24.

6 Shih IM, Kumaran RJ. Epitheloid trophoblastic tumor: a neoplasm distinct from choriocarcinoma and placental site trophoblastic tumor simulating carcinoma. Am J Surg Pathol 1998;22:1393-403.

7 Shih IM, Kumaran RJ. p63 expression useful in the distinction of epithelioid trophoblastic and placental site trophoblastic tumors by profiling trophoblastic subpopulations. Am J Surg Pathol 2004;28:1177-83.

8 Knox S, Brooks SE, Wong-You-Cheong J, et al. Choriocarcinoma and epithelial trophoblastic tumor: successful treatment of relapse with hysterectomy and high-dose chemotherapy with peripheral stem cell support: a case report. Gynecol Oncol 2002;85:204-8. 